\title{
Percepción sobre servicios ecosistémicos culturales asociados al bosque nativo por parte de un grupo universitario de estudiantes de pedagogía
}

\section{Perception on cultural ecosystem services associated with the native forest by a university group of students of pedagogy}

Percepção por parte de um grupo de estudantes universitários de pedagogia dos serviços ecossistêmicos culturais associados à floresta nativa

Cristián Aguilar-Correa Universidad Católica del Maule

Talca, Chile caguilar@ucm.cl

http://orcid.org/0000-0002-2639-7216

Camila Valencia-Fuentes Universidad Católica del Maule

Talca, Chile

camilavalencia.1234@gmail.com

http://orcid.org/0000-0001-9899-010X

Maite Huentemilla-Rebolledo

Universidad Católica del Maule

Talca, Chile

maiteangelicahr@gmail.com http://orcid.org/0000-0003-4173-3481

Daniela Valderrama-González

Universidad Católica del Maule

Talca, Chile

danielavalderrama@live.cl

http://orcid.org/0000-0002-7076-2620

Ángela Rojas-Correa Universidad Católica del Maule

Talca, Chile

anparoco@gmail.com

http://orcid.org/0000-0003-0853-4800

Mackarena Méndez-Contreras

Universidad Católica del Maule

Talca, Chile

mendezmackarena@gmail.com

http://orcid.org/0000-0001-7353-604X

Carla Tapia-Hernández Universidad Católica del Maule

Talca, Chile

tapia.carlavaleska@gmail.com

http://orcid.org/0000-0002-8129-341X

Recibido • Received • Recebido: 28 / 12 / 2017

Corregido • Revised • Revisado: 11 / 03 / 2019

Aceptado • Accepted • Aprovado: 02 / 06 / 2019 
doi: http://dx.doi.org/10.15359/ree.23-3.19

URL: http://www.una.ac.cr/educare

CORREO: educare@una.cr

Resumen: La investigación tuvo por objetivo analizar las percepciones que posee un grupo de 15 docentes en formación inicial respecto de los servicios ecosistémicos culturales asociados al bosque nativo. Los sujetos participantes fueron estudiantes de las carreras de Pedagogía en Educación General Básica, ingresos 2013, 2014, 2015, 2016 y Pedagogía en Educación General Básica con Mención, ingreso 2017, de la Universidad Católica del Maule-Chile. En el estudio se emplearon dos técnicas de trabajo cualitativas, el grupo focal y el dibujo identitario revisado. Los resultados arrojan que en docentes en formación coexisten una serie de visiones alternativas respecto de los servicios ecosistémicos, que relacionan con temáticas de reciclaje e instituciones gubernamentales, pero también se evidencia la importancia que los sujetos participantes asignan a los innumerables beneficios y aportes que entregan los servicios ecosistémicos al ser humano, así como también, la trascendencia que tienen los bosques nativos y sus servicios ecosistémicos culturales, como espacios pedagógicos al servicio de aprendizajes transversales en la formación del alumnado de enseñanza básica.

Palabras claves: Percepciones; educación; formación de docentes; ecosistema; bosque.

Abstract: This research aimed to analyze the perceptions that have a group of 15 teachers in initial training regarding the cultural ecosystem services associated with the native forest. The participants were students of the careers of Pedagogy in Basic General Education (generations of 2013, 2014, 2015, 2016), and of Pedagogy in primary education with an honorable mention (the generation of 2017) from the Universidad Católica del Maule-Chile. Two qualitative working techniques used in the study were the focus group and the revised identity drawing. The results showed that in pedagogy students alternative visions coexist with respect to ecosystem services; the participating students relate these services to recycling topics and government institutions. The results also showed the importance that participants assign both to the innumerable benefits and contributions that ecosystem services deliver to human beings and to native forests and their cultural ecosystem services as pedagogical spaces at the service of transversal learning in the development of basic education students.

Keywords: Perceptions; education; teacher training; ecosystem; forest.

Resumo: O objetivo da pesquisa foi analisar as percepções de um grupo de 15 estudantes de pedagogia em fase inicial sobre os serviços ecossistêmicos culturais associados à floresta nativa. Os participantes foram estudantes das carreiras de Pedagogia na Educação Geral Básica, iniciado em 2013, 2014, 2015, 2016 e Pedagogia na Educação Geral Básica com Menção, iniciado em 2017, da Universidade Católica de Maule-Chile. Duas técnicas qualitativas de trabalho foram utilizadas no estudo: o grupo focal e o desenho de identidade revisado. Os resultados mostram que nos professores em etapa de formação coexistem uma série de visões alternativas sobre serviços ecossistêmicos, que se relacionam com questões de reciclagem e instituições governamentais, mas também é evidente a importância que os sujeitos participantes atribuem aos inúmeros benefícios e contribuições que fornecem serviços ecossistêmicos aos seres humanos, bem como a importância das florestas nativas e seus serviços ecossistêmicos culturais, como espaços pedagógicos ao serviço da aprendizagem transversal na formação de estudantes da educação básica.

Palavras-chave: Percepções; educação; formação de professores; ecossistema; floresta. 


\section{Introducción}

En los últimos años, se observa un aumento de las investigaciones y publicaciones que hacen referencia a los servicios ecosistémicos, no solo en contextos ecológicos y medioambientales, sino también desde otras esferas del quehacer humano; llámese político, económico, cultural, educativo, etc. (Bitrán, 2015; Brea, 2014; Castillo y Almonacid, 2012).

Se entienden, por servicios ecosistémicos, todos los beneficios, tanto materiales como no materiales que se pueden obtener de los ecosistemas naturales (Alcamo et al. 2003).

Ahora bien, desde el punto de vista formativo, se podría argumentar que la temática en cuestión está de una u otra manera prescrita en cada currículo educativo; sin embargo, el trabajo didáctico referido a servicios ecosistémicos culturales es un área de incipiente desarrollo en la escuela y sobre la cual todavía no existe suficiente claridad de cómo abordarla.

La labor docente es trascendental frente a los aprendizajes y las experiencias formativas de sus estudiantes. Un desarrollo holístico del alumnado, sobre todo en la enseñanza básica, dependerá de una u otra forma, de las experiencias de enseñanza vividas y de los significados que construyan durante su itinerario formativo. Por tanto, los entornos didáctico-pedagógicos generados, sean estos dentro o fuera del aula, serán clave en este reto pedagógico.

Con estos propósitos en mente, quisimos llevar a cabo esta investigación. Nuestro objetivo, poder analizar y describir las visiones que posee un grupo de futuro personal docente de enseñanza básica sobre la temática en cuestión y, al mismo tiempo, poder vislumbrar el valor pedagógico que le otorgan a estos territorios como futuros maestros y maestras.

Finalmente, a partir de estas visiones, el personal docente modela y guía su labor y, al mismo tiempo, condiciona los acontecimientos que ocurrirán en el aula (García-Carmona, Vásquez y Manassero, 2011). Por tanto, comprender la transformación de una visión, de un saber no solo requiere de una reestructuración del armazón didáctico en su totalidad, sino también, de una reestructuración epistemológica, la cual admite un enorme desafío para las universidades que hoy forman profesores y profesoras.

\section{Referentes teóricos}

El concepto de servicios ecosistémicos comenzó a tomar mayor relevancia en la década de los ochenta, pero su inicio se remonta a la denominada era industrial. Este concepto tiene diferentes acepciones, todas ellas con perspectivas de análisis, a veces, un tanto diversas. Caro-Caro y Torres-Mora (2015) indican que a finales del siglo XX e inicios del XXI se impulsó el concepto de servicios ecosistémicos definidos como un amplio rango de condiciones y procesos a través de los cuales los ecosistemas naturales y las especies que hacen parte de ellos ayudan a sostener la vida humana. 
doi: http://dx.doi.org/10.15359/ree.23-3.19

URL: http://www.una.ac.cr/educare

CORREO: educare@una.cr

Por su parte, De Groot, Wilson y Bounmas (2002) sostienen que los servicios ecosistémicos son las funciones del ecosistema, donde la capacidad de los procesos y componentes naturales son los que proporcionan bienes y servicios que satisfacen las necesidades humanas, directa o indirectamente. No existe una definición totalmente aceptada o una clasificación base a partir de las cuales se puedan valorar, integralmente, los servicios del ecosistema.

Finalmente, Boyd y Banzhaf (2007) se refieren a los servicios ecosistémicos como:

Los componentes de la naturaleza, disfrutados, o directamente usados para bienestar humano. Siendo así, únicamente aquellos procesos ecológicos que se incorporan en la producción de los productos y servicios que usa la gente. Es decir, se procura separar los servicios ecosistémicos que surgen o están disponibles por la acción de la naturaleza, de los beneficios que la sociedad obtiene de ellos a partir de alguna acción o intervención específica. (p. 620)

Las definiciones concuerdan en el uso que se le da por parte de los seres humanos a los servicios ecosistémicos. La naturaleza, indiscutiblemente, aporta de manera directa o indirectamente beneficios y servicios indispensables para el desarrollo de la vida en el planeta.

De acuerdo con el informe sobre Ecosistemas y bienestar humano de la evaluación de Alcamo et al. (2003), los servicios ecosistémicos se pueden clasificar de manera general en cuatro categorías: "servicios de suministro, regulación, de base y culturales" (p. 9).

Respecto de los servicios ecosistémicos culturales, estos corresponderían a aquellos servicios que prestan los ecosistemas y que se relacionan con la valoración humana no material de los espacios naturales (Figueroa, 2010).

De Groot et al. (2002), al respecto, señalan que los servicios culturales son el resultado de la evolución a lo largo del tiempo y del espacio de la relación entre los seres humanos y la naturaleza que los rodea; como resultado, las culturas humanas están muy influenciadas por los ecosistemas que habitan y viceversa. Los servicios culturales abarcan tanto los aspectos materiales (las plantas, los animales, el agua, el suelo) como los aspectos no materiales (seguridad, belleza, espiritualidad, recreación cultural y social para las poblaciones); también abarcan el conocimiento, las percepciones y los sistemas de clasificación de su entorno natural.

Dentro de los bienes intangibles de los ecosistemas declarados por Alcamo et al. (2003), podemos mencionar beneficios espirituales y religiosos, recreación y ecoturismo, estéticos, de inspiración, educacionales, sentido de identidad y pertenencia a un lugar y herencia cultural. 
Los momentos de reflexión y conexión, que entrega un entorno natural, son cada vez más difíciles de encontrar; sin embargo, son estos espacios fuente vivificante de autorreflexión y búsqueda constante de nuevos horizontes estéticos. La naturaleza, en este sentido, es un elemento clave en nuestro desarrollo global. La complejidad, estética y dinamismo de los ecosistemas boscosos, necesariamente, debe hacernos transitar desde un paradigma antropocéntrico a otro biocéntrico.

Ahora bien, para algunas sociedades, la conservación de un bosque con sus especies endémicas y nativas puede ser considerado con un valor fundamental. Sin embargo, para otras, un bosque nativo significa un obstáculo para el desarrollo y el crecimiento económico (Barrera, Gómez-Pompa y Vázquez-Yanes, 1977). Los bosques nativos, recurrentemente, han sido considerados como una fuente inagotable de recursos económicos. Es importante señalar, que los servicios y beneficios que los bosques nativos entregan a la sociedad van mucho más allá de productos tangibles, también se encuentran los servicios culturales (beneficios no materiales), como la recreación, actividad turística y espiritual, además del importante valor cultural que el bosque nativo entrega a comunidades indígenas y campesinas.

Los bosques nos ofrecen valores intrínsecos que escapan de la concepción materialista donde solamente se valora aquello que es tangible y puede ser utilizado. En esta categoría, se considera esencialmente el valor de existencia (Bitrán, 2015).

Por otra parte, al realizar un análisis exhaustivo del currículo chileno para la enseñanza básica, encontramos objetivos de aprendizaje que abordan los servicios ecosistémicos y el bosque nativo; no obstante, se plantean principalmente desde una perspectiva declarativa y conceptual. Al respecto Sanmartí (2002) indica:

Durante mucho tiempo, los profesores de educación básica se concentraron en realizar una enseñanza más conceptual, centrada en la transmisión de conocimientos descriptivos, que promovía el trabajo de laboratorio o de actividades experimentales excluidas de la teoría misma y asociadas a una visión distorsionada de la ciencia con un método único. (p. 23)

Finalmente, es fundamental subrayar la importancia de favorecer en el estudiantado una conciencia ecológica con su entorno, al desarrollar valores y actitudes positivas hacia los bosques nativos. "Las enseñanzas de estas competencias deben hacerse de manera integrada en el currículo, deben formar parte del proceso enseñanza-aprendizaje y no de manera aislada" (Alagarda, 2015, p. 15). Lograr esto es fundamental en la formación inicial docente. Cuando un profesor o profesora poseen conciencia ecológica, sus acciones pedagógicas se ven reflejadas tanto dentro del aula como fuera de esta. 
doi: http://dx.doi.org/10.15359/ree.23-3.19

URL: http://www.una.ac.cr/educare

CORREO: educare@una.cr

\section{Marco metodológico}

\section{Diseño}

El diseño utilizado fue transseccional descriptivo, fundamentado desde un enfoque cualitativo.

\section{Participantes}

Las personas participantes son estudiantes regulares de la carrera de Pedagogía en Educación General Básica y estudiantes del primer año de la carrera de Pedagogía en Educación General Básica con mención de la Universidad Católica del Maule-Chile. Del grupo 11 fueron mujeres (73.3 \%) y 4 (26.7\%) hombres. Las edades fluctuaban entre los 18 y 40 años.

Respecto de algunas consideraciones éticas y organizativas, dada la naturaleza de la investigación, cabe señalar que las personas participantes inicialmente, y de manera libre y voluntaria, firmaron un consentimiento informado en donde autorizaban la grabación y transcripción de las respuestas emitidas durante el desarrollo del grupo focal, como también, otorgar facultades al equipo investigador para publicar los dibujos llevados a cabo.

Finalmente, y a objeto de facilitar el análisis e interpretación de los datos y mantener el anonimato del grupo de participantes, se establecieron códigos informativos. El ordenamiento respectivo considera el grafema E que denomina la figura Estudiante y un valor numérico que designa correlato de colaboración durante el proceso. Ej. E5: corresponde a Estudiante número 5 en el estudio.

\section{Instrumentos}

En esta investigación se utilizaron dos instrumentos; el desarrollo de un grupo focal, a través de unas preguntas semiestructuradas, y la técnica del dibujo identitario revisado de Bagnoli (2004). La batería de preguntas que guiaron el dialogo fueron cinco, cada una de ellas enfocadas en la temática en cuestión y validadas por juicio experto (Lawshe, 1975). El propósito fue que docentes en formación lograran expresar de manera libre sus ideas y emociones; con esto se provoca una conversación que se enriquece con las opiniones de la totalidad de participantes y permite un diálogo efectivo y espontáneo. Cabe destacar que las respuestas obtenidas fueron grabadas para su posterior transcripción y análisis, y regidas por los siguientes procedimientos: método de comparación constante: Implicó generar teorías mediante un procedimiento analítico de comparación, desarrollando categorías (Glaser y Strauss, 1999) y 
una codificación: consistente en un microanálisis de información a objeto de extraer la esencia de los datos, elaborar conceptos, hipótesis, teorías y establecer relaciones entre estos mismos (Schettini y Cortazzo, 2015). Finalmente, y a partir de los flujos de información recabada, se crearon códigos cuyo detalle se explicita a continuación:

Preconceptos asociados a los servicios ecosistémicos

Contribución educativa de los ecosistemas naturales

Conceptos asociados al aprendizaje no material

Preservación de la naturaleza

Conocimiento vinculado al enriquecimiento espiritual

Prácticas pedagógicas enfocadas en los servicios ecosistémicos culturales

Importancia otorgada al enriquecimiento espiritual del estudiantado dentro del currículo nacional

El segundo instrumento utilizado fue el uso de la técnica del dibujo identitario. Este tipo de instrumento es utilizado para detectar fondos de conocimiento e identidad en los sujetos (Bagnoli, 2004; Esteban, 2012). La práctica consiste en solicitar a cada participante que desarrolle un dibujo sobre sí o bien sobre una temática específica para posteriormente realizar una explicación escrita de lo realizado. En nuestro caso, se les solicitó que llevaran a cabo un dibujo respecto de cómo se veían dentro de un bosque. Finalmente, a partir de los dibujos y los datos explicativos del grupo en estudio se levantaron 4 códigos que detallamos a continuación:

Relación ser humano y naturaleza

Elementos asociados al ecosistema

Sentido de pertenencia

Actividades de recreación 
doi: http://dx.doi.org/10.15359/ree.23-3.19

URL: http://www.una.ac.cr/educare

CORREO: educare@una.cr

\section{Análisis y discusión}

\section{Grupo focal}

\section{Preconceptos asociados a los servicios ecosistémicos}

Frente a la pregunta: ¿qué sabes acerca de los servicios ecosistémicos?, destacamos lo afirmado por E6: "... lo veo como las cosas que nos ofrece los distintos ecosistemas, ... los recursos naturales que podemos encontrar como las ventajas que permiten que el ser humano tenga vida, un aire apropiado etc.." Además, E7 comenta que "al pensar en servicios ecosistémicos no pienso en algo que usa el ser humano para cuidar a la naturaleza, sino que lo que la naturaleza nos brinda y nosotros utilizamos". Esta declaración se ajusta a lo beneficios que la naturaleza brinda por sobre lo que el ser humano puede otorgarle a ella. En contraparte destacamos lo mencionado por E5 "... guardaparques, y los que tienen que ver con los incendios ... los brigadistas". Asimismo, E3 menciona que los servicios ecosistémicos son las "organizaciones que se dedican a cuidar lo que es el medio ambiente, el ecosistema... podemos desprender las tres erres, o sea, como disminuir el consumo, reciclar, reducir, reutilizar, o sea algo así, es lo que yo entiendo".

Los preconceptos que han manifestado se significan desde sus experiencias previas, las cuales se han forjado en grupos de pertenencia y en un potente medio cultural que va irisando, de una u otra forma, los significados que los sujetos atribuimos a conceptos y entidades. Al respecto, Novo (2002) hace mención a cómo se producen los preconceptos y su relevancia con el entorno:

Las ideas y construcciones que producimos son fruto de una negociación entre la realidad que contemplamos y las [experiencias], deseos, preconceptos, que conforman nuestros esquemas provisores de conciencia, junto a nuestra propia historia, a lo que hay que añadir el valor de los contextos, las influencias ambientales. (p. 19)

\section{Contribución educativa de los ecosistemas naturales}

Frente a esta temática podemos destacar lo que dice E8:

El medio ambiente es como el recurso más factible que tenemos, por ejemplo, para realizar una clase perfectamente podemos ... utilizar todos esos recursos que nos entrega la naturaleza en clase de matemática, en lenguaje y en cualquier asignatura.

Es así como el profesorado en formación concuerdan en que los ecosistemas naturales son un beneficio para el aprendizaje del estudiantado, E4 expresa"... es el material concreto más eficaz que tenemos, el material concreto más valioso y válido que podemos utilizar nosotros como futuros profesores". Reforzando lo anterior E1 señala que: ... "Ios niños pueden aprenderlo todo en la naturaleza, entonces el aporte es muy amplio". 


\section{Conceptos asociados al aprendizaje no material}

Cuando nos referimos al aprendizaje no material, hacemos alusión al aprendizaje interno que puede adquirir el alumnado, es decir, aquel conocimiento que no es conceptual, y que se basa en actitudes y sistema de valores que desarrolla frente a diversas temáticas. Considerando la importancia de los aprendizajes no materiales, debemos tener en cuenta que desde los ecosistemas se pueden obtener valores intrínsecos, los cuales no son meros aprendizajes conceptuales que normalmente suceden en las salas de clases, en esta categoría se considera, principalmente, el valor de existencia (Bitrán, 2015).

En este código destacamos lo dicho por E1 "... por ejemplo, algo que no se va a ver plasmado en una nota o en algo así como concreto, ... algo como más espiritual". Fortaleciendo lo anterior, E11 menciona que los aprendizajes no materiales son: "... algo interior, obviamente algo que uno puede conseguir con uno mismo, quizás también algo que se pueda compartir". Al respecto E7 señala, "... el aprendizaje no material es lo que se ve reflejado en los OAT (Objetivos Aprendizaje Transversales) y que no solamente se dan en el aula como dice claramente los objetivos transversales, sino que también está relacionados con toda la sociedad, o sea del colegio, todos los agentes".

\section{Preservación de la naturaleza}

Este código surge a partir de las preguntas: ¿qué sabes acerca de los servicios ecosistémicos? y como futuro profesor o profesora de enseñanza básica, ¿qué aprendizajes desarrollarías con tus estudiantes al visitar un bosque nativo? Para comenzar el análisis, recordamos lo mencionado por Sukhdev (2008), quien señala: "no hay que olvidar que la evolución del ser humano ha estado determinada por el medio ambiente, y que esta relación con la naturaleza tiene una gran importancia social, cultural y estética" (p. 12). Al reconocer que la relación del ser humano con la naturaleza tiene un gran valor, se manifiesta con mayor fuerza la necesidad de preservarla.

En el grupo focal, el grupo de docentes en formación desprenden de este código diferentes conceptos e ideas propias, entre ellas se encuentra la intención de preservar y mantener el bosque, se reconocen su propia existencia como un ser vivo que tiene el deber de cuidar la naturaleza. También indican que existen agentes oficiales que se dedican a la protección y el cuidado, pero no olvidan que cada persona, aunque no forme parte de alguna organización, se debe hacer responsable de esta labor. Podemos reafirmar lo dicho anteriormente, con las palabras del grupo participante. Con respecto a la preservación de la naturaleza, E5 señala: “... algún servicio que tiene que ver con proteger a la flora y fauna que está en peligro extinción ....". Interpreta que la preservación de la naturaleza no solo tiene que ver con los árboles, sino que con toda manifestación de vida. Al ser docentes en formación, tienen en cuenta que van a formar personas, y consideran esencial poder trabajar, en el futuro, con sus estudiantes la importancia de preservar aquello que nos rodea: "... mantener ese bosque, eso tan maravilloso que vieron, a partir 
doi: http://dx.doi.org/10.15359/ree.23-3.19

URL: http://www.una.ac.cr/educare

CORREO: educare@una.cr

de ello empezar a enseñar contenidos, como empezar a relacionarlos para poder preservar y poder mantener el bosque" (E1). La importancia de este código radica, principalmente, en lo publicado por Ávila (2011): "no se puede desintegrar el concepto de ser humano con la naturaleza, ambos son uno, de ahí que hacer daño a la naturaleza es hacerse daño a sí mismo" (p. 211).

En Chile, el Estado tiene la función de tutelar la conservación de la naturaleza dentro del artículo $19^{\circ}$, número $8^{\circ}$, el que consagra como derecho el coexistir en un medio ambiente libre de contaminación (Constitución Política de la República de Chile, 1980).

\section{Conocimientos vinculados al enriquecimiento espiritual}

El estudiantado afirman que el enriquecimiento espiritual desarrolla diferentes habilidades, sean estas sociales, intelectuales y reflexivas. Valora la contribución que tiene la naturaleza para el desarrollo del ser humano. Como dice E11 frente al enriquecimiento espiritual "Yo creo que es algo que te llena, es como algo interior". Complementando esto, podemos destacar lo dicho por E7 "... I a naturaleza contribuye en todo aquello en que la teoría no puede llegar o sea la práctica y que cómo decir que cualquier actividad que nosotros nos propongamos podemos llevarlos a la naturaleza". Al respecto, Castillo y Almonacid (2012) señalan:

... por medio de los sentidos que se logra llegar a la sensación, de ella a la emoción y de ahí a los sentimientos, para luego sumergirse en la conciencia, de la cual cada individuo precisa en su proceso personal y profesional como fruto de un medio cultural que lo impregna, de una praxis que lo caracteriza y de un estilo que lo distingue como único. A partir de esta perspectiva, se logra comprender cómo los sentidos traspasan las definiciones unidireccionales, referidas, por un lado, a los clásicos conocidos como vista, oído, tacto, gusto y por otro, a los sentidos entendidos desde el significado de existencia, a partir de las propias vivencias (p.669).

\section{Prácticas pedagógicas enfocadas hacia los servicios ecosistémicos culturales}

Surasky y Morosi (2013) indican la realidad del ser humano en relación con el valor que le entregan a la naturaleza y señalan:

Es posible y necesario desandar el camino que ha llevado a dar preeminencia a la naturaleza como fuente de riquezas por sobre la naturaleza como vida, es indispensable desarmar la madeja que creó ajenidad entre los seres humanos y el entorno natural. (p. 9) 
Ante la pregunta:como futuro profesor o profesora de enseñanza básica, ¿qué aprendizajes desarrollarías en tus estudiantes al visitar un bosque nativo? El profesorado en formación ha señalado sus ideas y convicciones. E2 expresa que "... cuando yo era chica una inspectora me dijo... yo estaba tirando unas ramas, sacándole las hojas a un árbol y ella me dijo no, no se lo hagas porque le duele, ¿te gusta a ti que te tiren el pelo? Entonces enseñarles no con una acción que yo le vaya a tirar el pelo a un niño, pero sí enseñarles que ellos también sienten tal cual como sentimos nosotros. Si le tiras un papel a un árbol o haces tus necesidades, ¿a ti te gustaría que te lo hicieran? La idea es que sienta tal como podría sentir un árbol o una planta, que se ponga en el lugar del otro, que sea empático". A través de la experiencia de E2 vemos lo relevante que puede llegar a ser una práctica pedagógica a temprana edad, lo que fue significativo para ella lo ha preservado en sus esquemas mentales posteriores.

Los niños y las niñas sienten una curiosidad innata por la naturaleza, la cual debe ser guiada por sujetos adultos más capacitados. Este aprendizaje guiado puede ser para bien o para mal, tal como sugiere E3 "Al llevar a los niños a visitar un bosque nativo primero que todo es un aprendizaje transversal, para valorar el entorno natural, saber que hay bosques nativos endémicos que ese árbol que tú ves no va a estar en ninguna parte del mundo porque es endémico, solamente está en la séptima región, por lo tanto el niño va a saber que hay que cuidarlo, porque si él no lo cuida, si su hermano chico no lo cuida, si cuando él salga de vacaciones con su papá y su papá quisiera cortar un rama de árbol para hacer fuego él le dirá que no, va saber que si no lo cuida va a desaparecer esa especie. Decirles que este bosque no solo tiene árboles, arbustos también tiene fauna hay pajaritos que sólo habitan estos bosques, hay animales que solamente se alimentan de estos frutos entonces darle un sentido de pertenencia a nuestro entorno natural". En relación con lo dicho anteriormente con las prácticas pedagógicas, es necesario vincular, en todo momento, la importancia de los servicios ecosistémicos culturales, tal como lo expresa E5: "Yo articularia igual todos los conocimientos con las actitudes, por ejemplo si los llevara a un bosque en la parte de conocimiento explicarles la diversidad de especies que existen, averiguar el nombre y la cantidad de años que tiene un árbol, la reproducción de ciertos animales, pero si todo debe ir de la mano con las actitudes. La actitud sería la actitud positiva y psicológica. La importancia que tiene ese bosque en nuestras vidas. Además, debemos inculcarles a los niños el proceso de cambio". Al respecto, Reimers (2003) comenta la importancia de buenas prácticas pedagógicas en la vida de los niños:

Los buenos maestros ... son [quienes] pueden situar su práctica en el contexto de las necesidades y perspectivas de esos estudiantes concretos. Son quienes pueden no sólo presentar contenidos educativos con claridad y [de manera] que los estudiantes puedan integrarlos al conjunto de significados que forman parte de su experiencia, sino que ... [atienden a estos] estudiantes como personas integrales, comprendiendo y [tomando en cuenta] sus necesidades de desarrollo emocional y social, [les ayuden] explícitamente a construir elevadas aspiraciones sobre su propio futuro. (p. 21) 
doi: http://dx.doi.org/10.15359/ree.23-3.19

URL: http://www.una.ac.cr/educare

CORREO: educare@una.cr

El personal docente en formación indica que enseñarle al estudiantado que los servicios ecosistémicos aportan mucho más que algo material es un trabajo colaborativo que tiene su inicio en el hogar y que los cuerpos docentes son los responsables de reforzarlo y acrecentarlo en el contexto escolar.

\section{Importancia otorgada al enriquecimiento espiritual del estudiantado desde el currículum nacional}

Estecódigo tiene relación con todos los otros que han surgido para el análisis,y se desprende de la pregunta: ¿crees que el sistema educativo actual fomenta el enriquecimiento espiritual del estudiantado?, las personas participantes del grupo focal han podido reflexionar a partir de sus experiencias en los distintos centros de práctica donde han evidenciado la realidad educativa frente a la temática. Es importante destacar lo manifestado por Coll y Solé (1987), quienes señalan que "con frecuencia, los contenidos escolares son asimilados a sistemas conceptuales, considerándose en ese caso que las actitudes, normas y valores que indefectiblemente vehicula la educación forman parte de una especie de 'currículum oculto'" (p. 80).

Al declarar que los contenidos se asimilan a sistemas conceptuales, se antepone la obtención de contenidos por sobre el enriquecimiento espiritual del estudiantado. Entre las respuestas obtenidas, podemos afirmar lo anterior con lo expuesto por E1: "... no les enseñan a los niños el valor de la naturaleza, sino que les enseñan procesos de la naturaleza como temas simplemente conceptuales que después se los van a evaluar en una prueba". Opinión que es compartida por otro docente en formación quien declara "... creo que el sistema no fortalece el enriquecimiento espiritual porque se le enseña más para pruebas estandarizadas entonces no se le enseña lo espiritual" (E8).

Por otro lado, hay docentes en formación que han experimentado una realidad distinta, y señalan que el currículo nacional fomenta el enriquecimiento espiritual: "Mira, con lo que he visto últimamente igual se ha promovido" (E14). Esa afirmación puede estar justificada a través de lo que señala otro docente en formación: "Yo creo que el sistema si lo permite, pero las instancias no se dan porque como decían por el contexto o por los mismos profesores que algunos tienen una mentalidad más cerrada, otros son más pragmáticos y no son muy llevados a la práctica".

Podemos concluir que el profesor tiene la facultad de otorgarle un sentido distinto a la educación, logrando que el estudiantado deje en segundo plano el aprendizaje para una prueba y tomen conciencia de la importancia que tiene formarse para la vida. Para finalizar citamos a E3, quien señaló"... ojalá que de niños nos enseñaran a luchar por ideales y no por calificaciones, así de adultos trabajaríamos por sueños y no por quincenas".

12 Cristián Aguilar-Correa, Camila Valencia-Fuentes, Maite Huentemilla-Rebolledo Daniela Valderrama-González, Ángela Rojas-Correa, Mackarena Méndez-Contreras y Carla Tapia-Hernández 


\section{Dibujo identitario revisado}

\section{Relación ser humano y naturaleza}

Este código define el grado de conexión que tiene el estudiantado con la naturaleza, específicamente con el bosque nativo. Bifani (1997) afirma que existe una estrecha relación indivisible entre la naturaleza y el ser humano, es una interacción entre ambas entidades, la naturaleza necesita de la humanidad y sobre todo esta necesita de la naturaleza. A partir de lo anterior, resaltamos la explicación del dibujo realizado por E2, quien declara: "Cuando yo dibujé pensé en que todos somos libres y tenemos los mismos derechos de vida, ninguno es superior a otro en la naturaleza" (ver Figura 1).

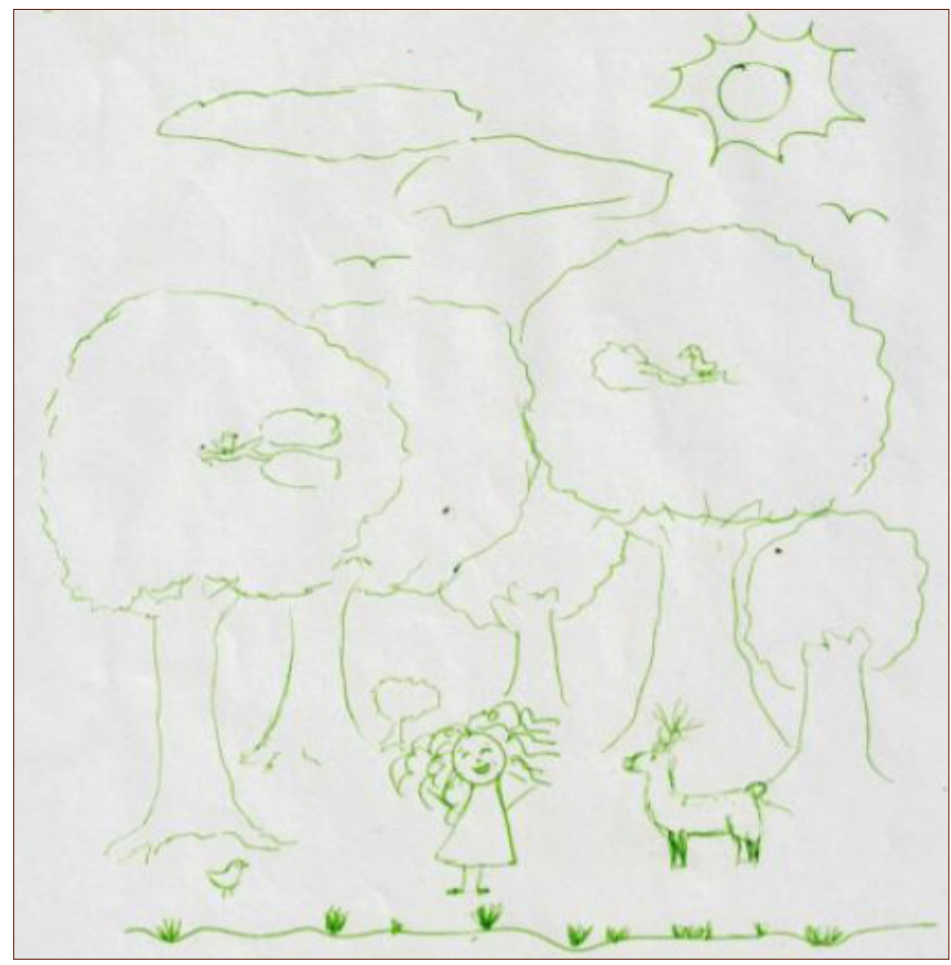

Figura 1: Dibujo identitario realizado por E2.

Otras personas del grupo consideran los bosques como algo aislado y alejado. Este es el caso de E10: "El aislamiento de todas las reservas naturales que están a lo largo del país, es por eso que está en una burbuja". E6 hace ver al bosque sin intervención humana "Me hice en un bosque lleno de montañas y árboles, porque es lo que me gusta, amo sentir la naturaleza así, sin la intervención del hombre" (ver Figura 2). 


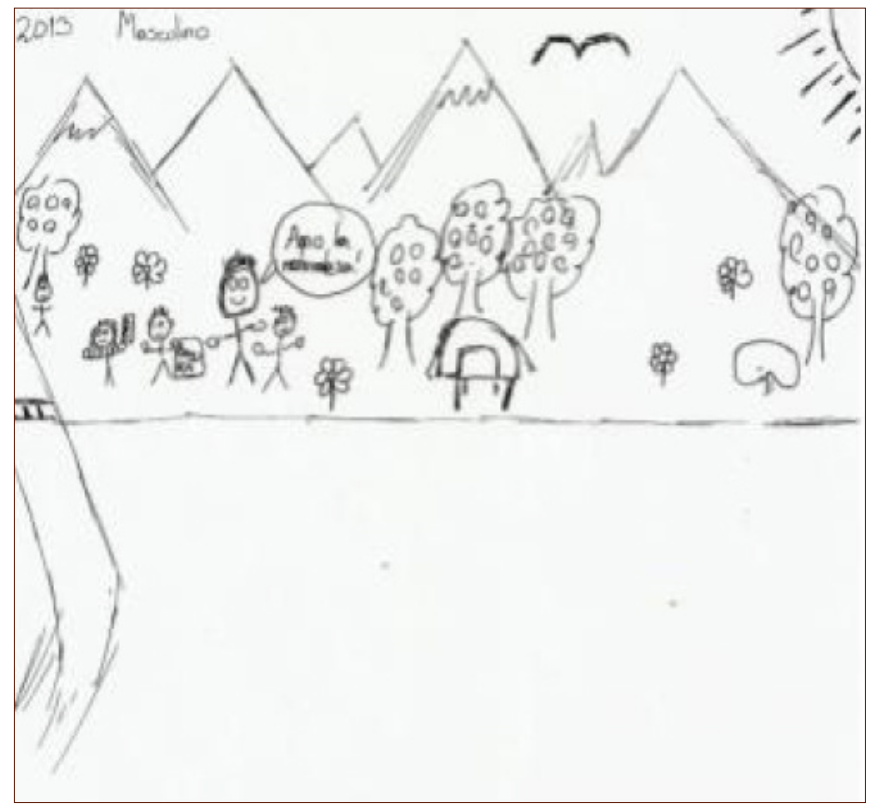

Figura 2: Dibujo identitario realizado por E6.

El personal docente en formación destaca en la explicación de los dibujos, aquellas actitudes que el ser humano debe tener dentro del bosque, a la vez, sienten al bosque como un lugar aislado y sin intervención humana. En este código, los dibujos coinciden en la presencia de árboles, animales y el sol, destacándose la forma en que cada protagonista se dibujó: con los brazos extendidos, lo que en sus relatos se expresa como sentimiento de libertad.

\section{Elementos asociados al ecosistema}

Al observar nuestro entorno, lo relacionamos con un sin fin de elementos que lo componen de manera directa como indirecta. Al respecto, Alcamo et al. (2003) se refiere a ecosistema como "... un complejo dinámico de comunidades de plantas, animales y microorganismos y el medio ambiente inorgánico que interactúan como una unidad funcional" (p. 3). Es decir, un ecosistema lo conforman un conjunto de seres vivos y elementos fisicoquímicos, los cuales están en constante interacción. "Cualquier lugar en el que se presenten estas condiciones podría considerarse como un ecosistema, sin importar el tamaño o lo breve que sea la duración de las interacciones" (Armenteras et al., 2016, p. 88).

De acuerdo con esto y teniendo en cuenta los elementos que conforman y permiten el adecuado funcionamiento de un ecosistema, planteamos lo recogido. E11 (ver Figura 3) menciona que "... dibujé agua porque asimilo mucho el bosque con el agua ... dibujé varios tipos de árboles". E5 complementa esto mencionando: "Me dibujérodeada de muchos árboles de todo tipo, ... Además, incorporé algunos animales... Dibujé rayos de sol entrando por medio de las ramas de los árboles y un riachuelo ya que siento el sonido del agua correr". 


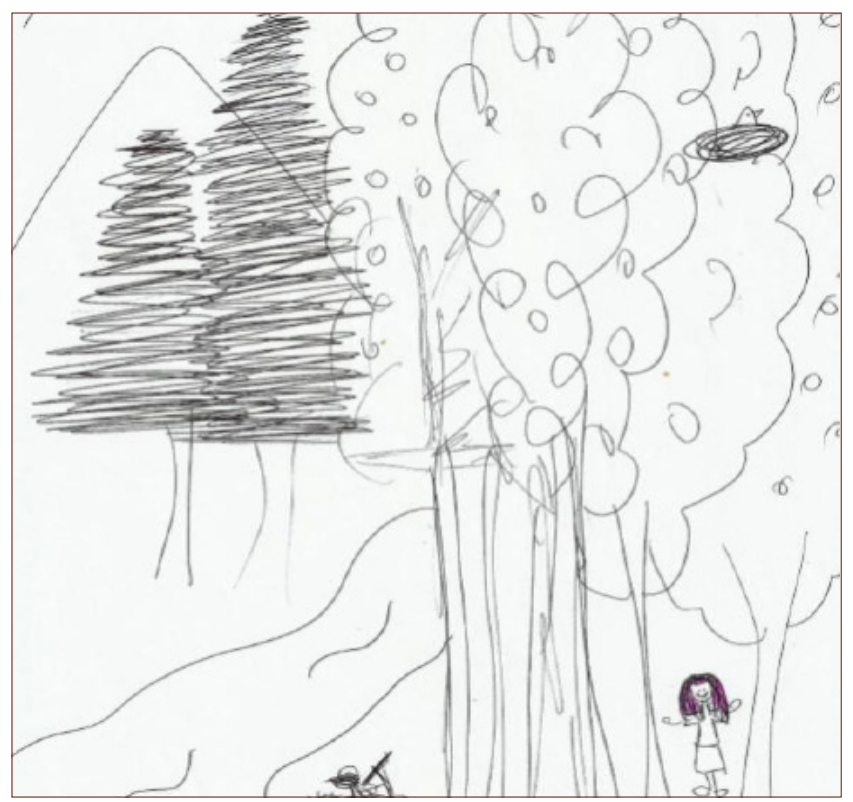

Figura 3: Dibujo identitario realizado por E11.

Las respuestas dadas por grupos de docentes en formación dejan entre ver que, para ellos, los elementos que conforman el ecosistema son cruciales a la hora de imaginarse en uno. A esto se suma la opinión de E12 quien en su dibujo se encuentra "... mirando un lago, ... escucha a los animales que salen a cazar". Adicionalmente menciona que está "... en un bosque de Araucarias". Se suma a ello E9 quien admira "... las especies y todo lo que tiene un bosque nativo" (ver Figura 4).

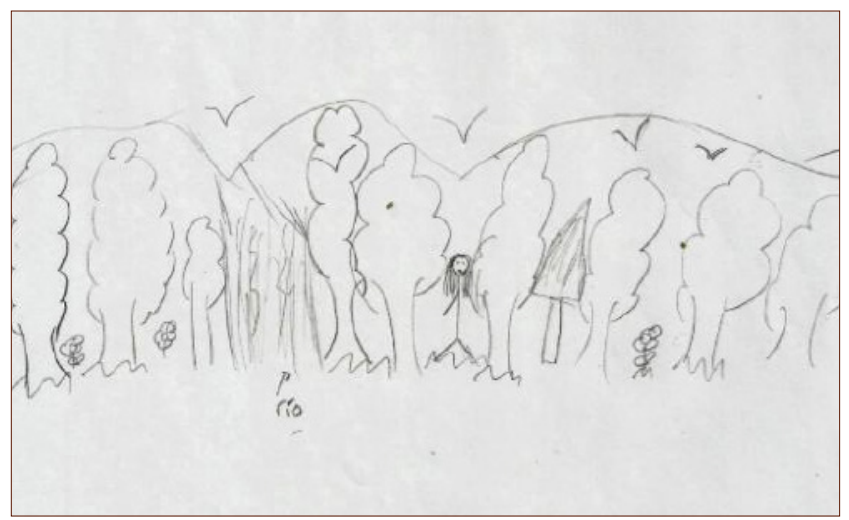

Figura 4: Dibujo identitario realizado por E9. 
doi: http://dx.doi.org/10.15359/ree.23-3.19

URL: http://www.una.ac.cr/educare

CORREO: educare@una.cr

Al analizar los elementos asociados al ecosistema que realiza el grupo de docentes en formación, señalamos la importancia que atribuyen a los elementos que conforman, en este caso, el bosque nativo. Informan que la presencia de diferentes tipos de árboles, zonas de relieve y fauna presente enriquecen el entorno y lo convierten en un ambiente ameno que les provoca sentimientos y emociones positivas. Por consiguiente, al observar los dibujos, se exterioriza un patrón con respecto a los elementos presentes en el bosque nativo, es decir, se observan factores bióticos como árboles, plantas y animales terrestres, acuáticos y aéreos. De la misma forma encontramos presentes factores abióticos como la luz (proveniente del sol), el agua (presente en nubes y ríos) y las montañas.

\section{Sentido de pertenencia}

Este código se desprende a partir de la visión del grupo participante en relación con el medio que les rodea y como consideran que forman parte de este. De los 15 dibujos obtenidos, 6 de ellos clasifican dentro del código "sentido de pertenencia", considerado como una pertenencia recíproca. Brea (2014) lo define así:

... sentimiento de identificación de un individuo con un grupo o con un lugar determinado. A partir de él emergen lazos afectivos que generan en la persona actitudes positivas hacia el grupo o el lugar. Entre estas se incluyen el deseo de participar en su desarrollo y la construcción de significados que luego formarán parte de su memoria personal y del grupo. (p. 9)

Según los estudios, el sentido de pertenencia provoca que las personas creen lazos de afectividad con aquellos lugares que las rodean, lo que les gusta, transformándolo en algo significativo para ellas. E1 nos comenta su fundamento sobre el dibujo: "Dibujé todo el bosque, y estoy yo al medio... dibujé montañas, animales, y se supone que yo estoy dentro del bosque como parte de este, no como un ser externo, ni como alguien que está botando basura o algo así, sino que tú eres parte del bosque. Eso es lo que dibujé yo" (ver Figura 5).

En el mismo margen, E7 comenta lo importante que es para ella el bosque: "Se supone que soy parte del árbol, ya que casi todos piensan que nosotros no somos parte de la naturaleza, como si estuviéramos en una categoría diferente, pero no, soy tanto árbol como soy persona" (ver figura 6).

Por último, E8 nos hace ver el rol que tiene el bosque, diciendo que: "La razón por la cual me dibujé tan pequeña entremedio del inmenso bosque es porque pienso eso, que la naturaleza es lo más importante y lo que debería destacar siempre. Que sea ella la protagonista de lo que es la vida y no los seres humanos. Que se les de la importancia que tiene y no desmerecerla" (ver Figura 7). 
doi: http://dx.doi.org/10.15359/ree.23-3.19

URL: http://www.una.ac.cr/educare

CORREO: educare@una.cr

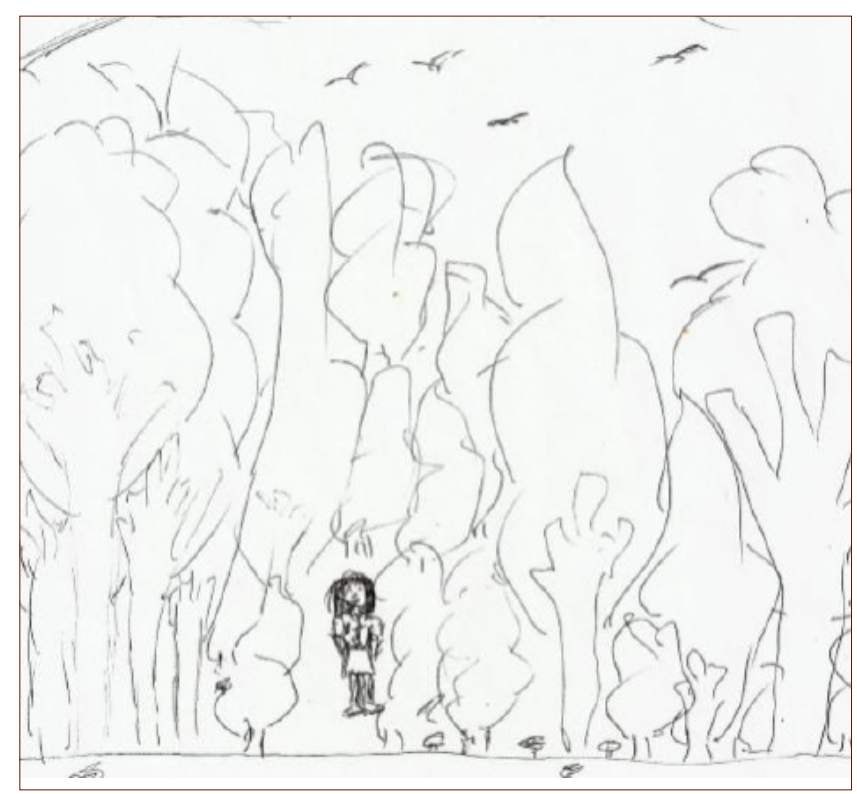

Figura 5: Dibujo identitario realizado por E1.

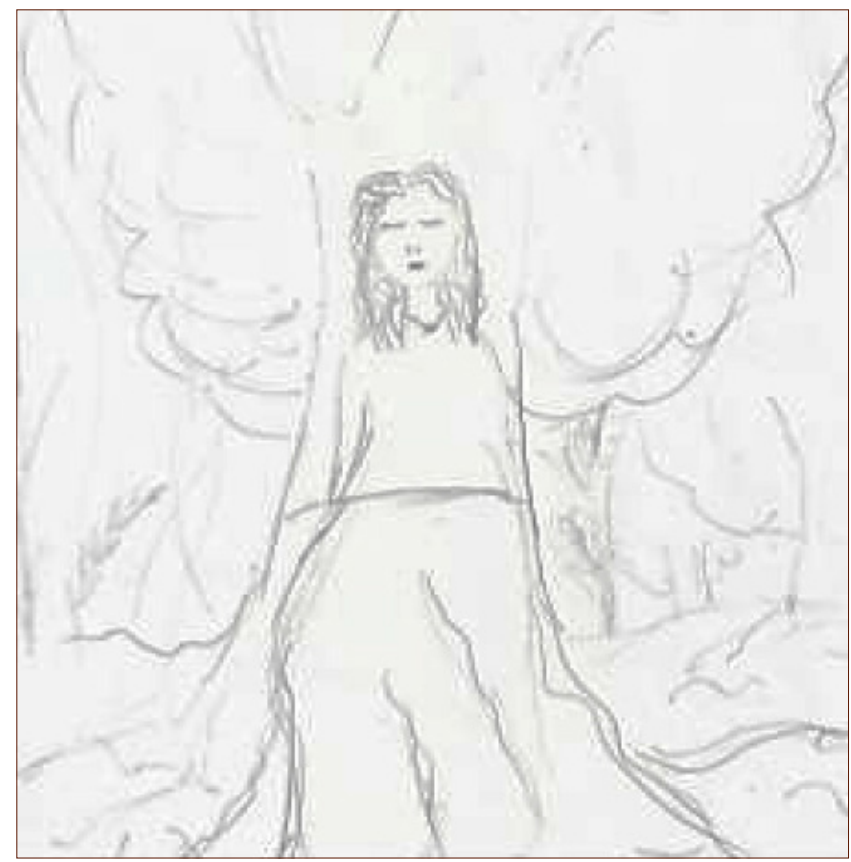

Figura 6: Dibujo identitario realizado por E7. 
doi: http://dx.doi.org/10.15359/ree.23-3.19

URL: http://www.una.ac.cr/educare

CORREO: educare@una.cr

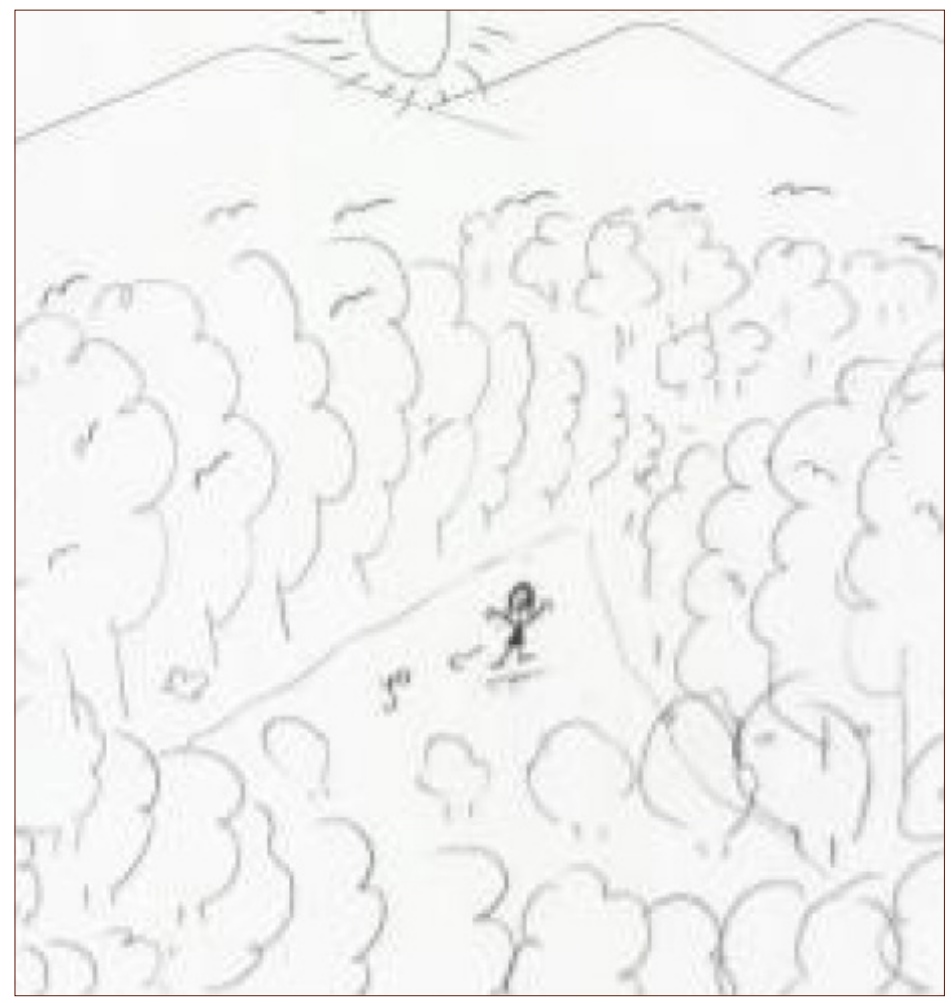

Figura 7: Dibujo identitario realizado por E8.

\section{Actividades de recreación}

En el último tiempo las actividades recreativas han marcado un antes y un después, puesto que la sociedad les entrega una mayor importancia. En general, lo que buscan los fines recreativos es el mejoramiento de la calidad de vida, logrando que las actividades sean placenteras. Además, esto permite que los individuos puedan desarrollar habilidades y sobre todo expresar emociones (Mateo, 2014). En esta misma línea, al observar y analizar lo explicado por el grupo de docentes en formación es necesario especificar que E9 comenta en relación a realizar actividades recreativas "... me permite desconectarme de todo y disfrutar de aquel paisaje que nos dan los dueños de casa que serían los árboles y animales presentes". Es notorio destacar la sintonía con la respuesta del docente en formación E9, con la de E15 quien señala que "... estando sola en un bosque lo que me gustaría hacer es leer un libro, es tranquila y relajante" (ver Figura 8). 


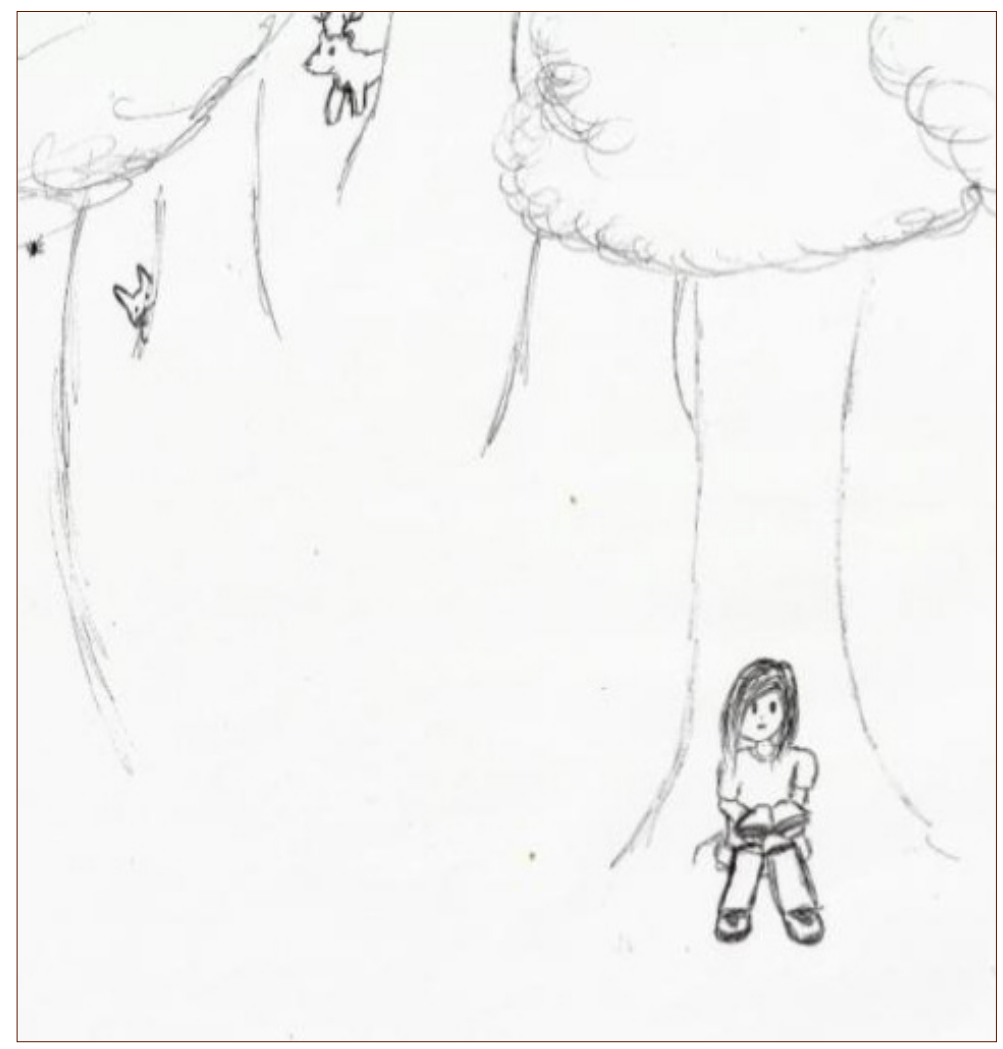

Figura 8: Dibujo identitario realizado por E15.

En momentos de ocio se puede realizar actividades recreativas, las cuales según Salazar (2008) deben ser:

... actividades agradables en las cuales se participa durante el tiempo libre y que fomentan el uso positivo de éste para promover el desarrollo integral de las personas. Este desarrollo integral se alcanza por medio de experiencias significativas de educación no formal, el disfrute de o el gozo por lo que se hace, la selección de actividades que agradan y la participación voluntaria en lo que se desea realizar. (p. 21)

En referencia con lo expuesto anteriormente, el sujeto E14 declara que: "... me gustaría casarme en un par de años, y me gustaría hacerlo en la naturaleza, ya que es preciosa." Esto deja en evidencia que el desarrollo de actividades recreativas en la naturaleza promueve experiencias positivas y significativas, que conllevan a un óptimo desarrollo integral del individuo (ver Figura 9). 
doi: http://dx.doi.org/10.15359/ree.23-3.19

URL: http://www.una.ac.cr/educare

CORREO: educare@una.cr

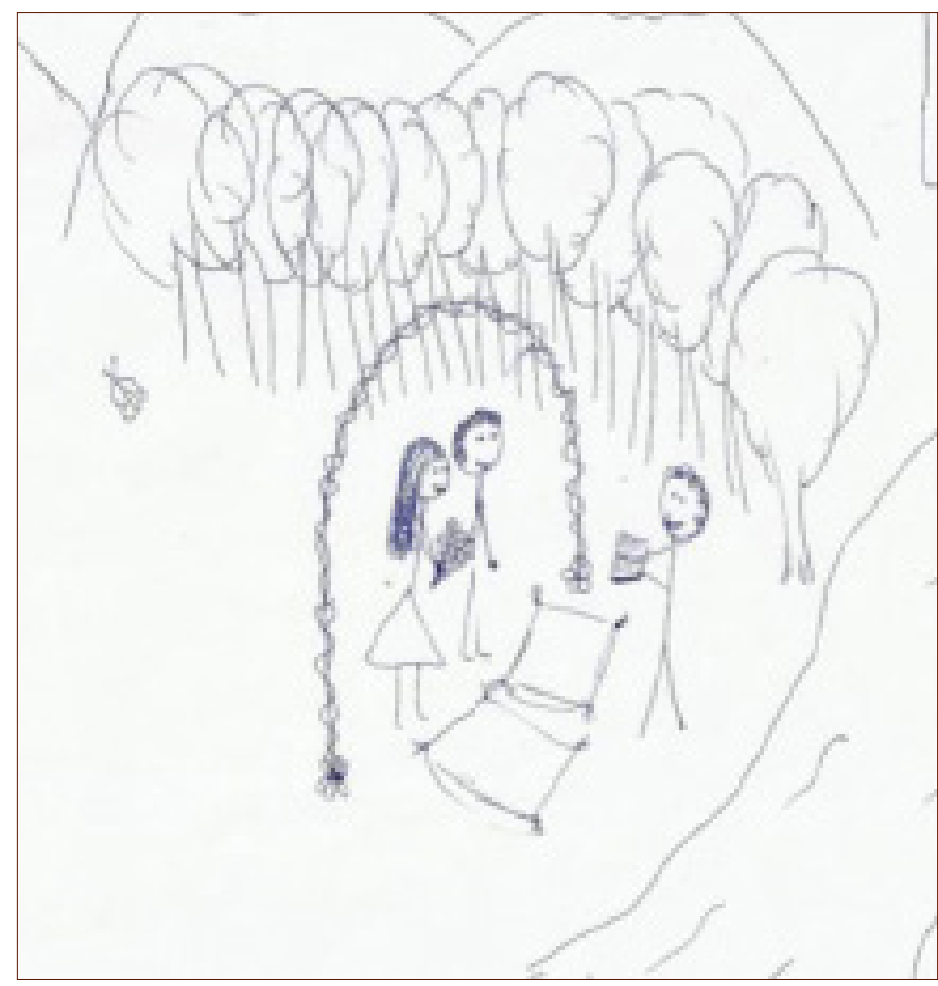

Figura 9: Dibujo identitario realizado por E14.

Las respuestas del estudiantado en formación están debidamente asociadas y arrojan que el disfrute y el estar en contacto con la naturaleza, les permite desconectarse y sobre todo lo ven como una actividad recreativa que les entrega felicidad. Al observar los dibujos asociados a las categorías de actividades recreativas, es posible identificar al individuo realizando diferentes acciones. Estas actividades favorecen a que puedan experimentar emociones y sensaciones en el ecosistema.

\section{Conclusiones}

La visión que poseen los profesores y profesoras en formación, respecto de los servicios ecosistémicos culturales, es diversa. Parte de la muestra tiene visiones alternativas, mayoritariamente asociadas a servicios de aprovisionamiento (suministros). Por el contrario, un grupo de estudiantes reconoce que los servicios culturales son aquellos servicios no materiales, fundamentales y necesarios en el desarrollo holístico del ser humano.

Por su parte, un porcentaje considerable de estudiantes le otorga gran importancia al concepto desde un paradigma principalmente medioambiental. De igual modo, manifiestan que estos servicios ecosistémicos están vinculados de manera directa con la perspectiva teórica ligada al ecologismo. Valoran, sobre todo, que el alumnado (escolar) reconozca la trascendencia actual del reciclado y reflexionen acerca de la regla de las tres erres (reducir, reutilizar, reciclar). 
También se evidencia una confusión conceptual, fundamentalmente en lo referente a sus funciones. Para el estudiantado participante, los servicios ecosistémicos están dados por las instituciones gubernamentales; son estas entidades las garantes, tanto del cuidado, como la protección y preservación de estos territorios.

Por otra parte, los profesores y profesoras en formación también dejan entrever que los servicios ecosistémicos culturales corresponden a aquellos beneficios intangibles que nos brinda la naturaleza, los cuales van más allá que lo puramente material. Hay visiones sobresalientes hacia aspectos cognitivos, afectivos y psicomotrices como elementos imprescindibles en el desarrollo global de los niños y las niñas.

El grupo de estudiantes participantes del estudio reconocen la importancia del bosque nativo como un escenario pedagógico de aprendizajes y saberes inmateriales, declaran que existe una formación orientada a la conservación y cuidado de los bosques nativos desde una perspectiva significativa y emocional. Sin embargo, también manifiestan la importancia de seguir trabajando estas temáticas en la escuela básica y relevan, en su persona, el desafío de abordarlo una vez ejerzan su labor como docentes. Igualmente, aluden a que la enseñanza de los servicios culturales se encuentra presente en el currículo nacional, pero ello no necesariamente implica valorarlos y trabajarlos en el proceso educativo cotidiano.

Por último, el estudiantado subraya que el proceso de enseñanza vinculado a los servicios ecosistémicos culturales es más trascendental y significativo cuando lo aprendido se realiza in situ.

Con respecto al dibujo identitario, el personal docente en formación otorga un gran valor al sentido de pertenencia e interdependencia con el medio natural. Los dibujos reflejan lo anteriormente descrito. El grupo participante se dibuja como parte consustancial de los ecosistemas y no como seres vivos periféricos. Atribuyen gran significado a aspectos relacionados con el disfrute y el desarrollo de las emociones. Del mismo modo, se evidencia que el personal docente en formación se dibuja libre... a este aspecto le entrega una gran connotación, puesto que señala que es en el único lugar donde puede expresar sus emociones, sentimientos y encontrarse consigo mismo.

Finalmente, no debemos olvidar que el conocimiento y el hacer pedagógico se ve condicionado por las perspectivas de las personas que los materializan, en nuestro caso profesores y profesoras de enseñanza básica. Su pensamiento, sus teorías, creencias y modelos sobre servicios ecosistémicos culturales guiarán su labor docente y condicionarán, de manera significativa y determinante, los acontecimientos que, al respecto, ocurrirán en el aula. 
doi: http://dx.doi.org/10.15359/ree.23-3.19

URL: http://www.una.ac.cr/educare

CORREO: educare@una.cr

\section{Referencias}

Alagarda, A. (2015). La importancia de gestionar las emociones en la escuela: Implicaciones en el proceso de enseñanza aprendizaje. Revista Supervisión, 36, 1-20. Recuperdo de http:// www.usie.es/SUPERVISION21/2015 36/SP 2136 Articulo La importancia de las emociones Alargarda.pdf

Alcamo, J., Ash, N., Butler, C. D., Callicott, J. B., Capistrano, D., Carpenter, S. R., ... Zurek, M. (2003). Ecosistemas y bienestar humano: Marco para la evaluación. Recuperado de https://goo.gl/ $\underline{\mathrm{K} 93 \mathrm{hZj}}$

Armenteras, D., González, T. M., Vergara, L. K., Luque, F. J., Rodríguez, N. y Bonilla, M. A. (2016). Revisión del concepto de ecosistema como "unidad de la naturaleza" 80 años después de su formulación. Ecosistemas, 25(1), 83-89. doi: https://doi.org/10.7818/ECOS.2016.25-1.12

Ávila, R. (2011). El derecho de la naturaleza: Fundamentos. En A. Acosta y E. Martínez (Comps.), La naturaleza con derechos. De la filosofía a la política (pp.173-238). Quito: Abya Yala.

Bagnoli, A. (2004). Researching identities with multi-method autobiographies. Sociological Research Online, 9(2), 1-15. doi: https://doi.org/10.5153/sro.909

Barrera, A., Gómez-Pomp, A. y Vázquez-Yanes, C. (1977). El manejo de las selvas por los mayas: Sus implicaciones silvícolas y agrícolas. Biótica, 2(2), 47-61. Recuperado de http://www. reservaeleden.org/agp/libro/lec/Cap23 manejo selvas.pdf

Bifani, P. (1997). Medio ambiente y desarrollo sustentable. Madrid: Red Almar.

Bitrán, D. (2015). Valoración de servicios ecosistémicos culturales para una zona desértica: La región de Tarapacá, Chile (Tesis de pregrado). Universidad de Chile, Santiago, Chile.

Boyd, J. y Banzhaf, S. (2007). What are ecosystem services? The need for standardized environmental accounting units. Ecological Economics, 63(2-3), 616-626. doi: https://doi. org/10.1016/j.ecolecon.2007.01.002

Brea, L. M. (2014). Factores determinantes del sentido de pertenencia de los estudiantes de arquitectura de la Pontificia Universidad Católica Madre y Maestra, Campus Santo Tomás de Aquino (Tesis de maestría). Universidad de Murcia, España. Recuperado de https://www. tdx.cat/bitstream/handle/10803/284952/TLMBA.pdf?sequence $=1$

Caro-Caro, C. I. y Torres-Mora, M. A. (2015). Servicios ecosistémicos como soporte para la gestión de sistemas socioecológicos: Aplicación en agroecosistemas. Orinoquia, 19(2), 237-252. doi: https://doi.org/10.22579/20112629.338 
Castillo, F. y Almonacid, A. (2012). Las actividades en la naturaleza en la formación inicial docente: Un acercamiento desde los sentidos. Educação e Pesquisa, 38(3), 667-681. Doi: http://dx.doi.org/10.1590/S1517-97022012005000012

Coll, C. y Solé, I. (1987). La importancia de los contenidos en la enseñanza. Recuperado de https:// goo.gl/pccXRa

Constitución Política de la República de Chile. (1980). Santiago, Chile: Editorial Jurídica Publigráfica. Recuperado de https://www.bcn.cl/obtienearchivo?id=document os/10221.1/60446/3/132632.pdf

De Groot, R., Wilson, M. y Boumans, R. M. J. (2002). A typology for the classification, description and valuation of ecosystem functions, goods and services. Ecological Economics, 41(3), 393-408. doi: https://doi.org/10.1016/S0921-8009(02)00089-7

Esteban, M. (2012). La multimetodología autobiográfica extendida y los fondos de identidad. Revista de Ciencias Sociales, 18(4), 587-600. Recuperado de https://www.redalyc.org/ html/280/28025469002/

Figueroa, E. (Consultor). (2010). Valoración económica detallada de las áreas protegidas de Chile. Santiago, Chile: Salesianos Impresores. Recuperado de http://bdrnap.mma.gob.cl/ recursos/privados/Recursos/CNAP/GEF-SNAP/Figueroa 2010.pdf

García-Carmona, A., Vázquez, Á. y Manassero, M. A. (2011). Estado actual y perspectivas de la enseñanza de la naturaleza de la ciencia: Una revisión de las creencias y obstáculos del profesorado. Enseñanza de las Ciencias, 29(3), 403-412. Recuperado de https://www.raco. cat/index.php/Ensenanza/article/view/247899/353583

Glaser, G. y Strauss, A. L. (1999). The Discovery of Grounded Theory: Strategies for qualitative research. Nueva York; Aldine de Gruyter.

Lawshe, C. H. (1975). A quantitative approach to content validity. Personnel psychology, 28(4), 536-565. doi: http://dx.doi.org/10.1111/j.1744-6570.1975.tb01393.x

Mateo, J. L. (2014). Las actividades recreativas: Sus características, clasificación y beneficios. EfDeportes.com, 19(196). Recuperado de https://www.efdeportes.com/efd196/lasactividades-recreativas-clasificacion.htm

Novo, M. (2002). Ciencia, arte y medio ambiente. Madrid: Editorial Paraninfo.

Reimers, F. (2003). Pedagogía, cultura escolar y formación de ciudadanía democrática en América Latina. Revista Latinoamericana de Estudios Educativos, 33(4), 9-50. Recuperado de https:// www.redalyc.org/articulo.oa?id=27033402 
doi: http://dx.doi.org/10.15359/ree.23-3.19

URL: http://www.una.ac.cr/educare

CORREO: educare@una.cr

Salazar, C. G. (2008). Recreación: Fuente inagotable de beneficios. En Foro centroamericano de recreación, educación física y deportes. Memoria del IV Congreso centroamericano de Educación Física, Deporte y Recreación "Naturaleza, movimiento y salud" (pp. 21-25). San José, Costa Rica: FECERED.

Sanmartí, N. (2002). Didáctica de las ciencias en la educación secundaria obligatoria. Madrid: Síntesis.

Schettini, P. y Cortazzo, I. (2015). Análisis de datos cualitativos en la investigación social: Procedimientos y herramientas para la interpretación de información cualitativa. Buenos Aires: Editorial de la Universidad de La Plata. Recuperado de https://eva.udelar.edu.uy/ pluginfile.php/1029065/mod resource/content/1/an\%C3\%A1lisis.pdf

Sukhdev, P. (2008). La economía de los ecosistemas y la biodiversidad. Cambridge, Reino Unido: Banson. Recuperado de http://www.teebweb.org/media/2008/05/TEEB-Interim-Report Spanish.pdf

Surasky, J. y Morosi, G. (2013). La relación entre los seres humanos y la naturaleza: Construcción, actualidad y proyecciones de un peligro ambiental. Documentos de Trabajo, 3, 1-38. Recuperado de http://www.iri.edu.ar/images/Documentos/noticias pagina web/ documentos de rebajo/doc trab 3 abril 2013.pdf 